\title{
Analysis of Design Parameters Influences on Modal Behaviour of Sandwich Panels
}

\author{
Aleksandar Vranićc ${ }^{1}$, Snežana Ćirić Kostićn \\ ${ }^{1}$ The Faculty of Mechanical and Civil Engineering in Kraljevo, University of Kragujevac, Serbia, vranic.a@ mfkv.kg.ac.rs
}

\begin{abstract}
The influences of various core configurations and other structural characteristics on vibrations of sandwich panels are discussed in the paper. The presented analysis contributes to efforts to resolve the contradiction between the requests for increased stiffness and reduced mass of mechanical structures. Additive manufacturing (AM) technologies enable manufacturing of complex lattice and cellular structures and design of optimal sandwich structures that satisfy both requests. Effects of each design parameters of a sandwich panel (core shape, wall thickness of plates and core, width of core) can be varied independently of the other parameters, thus contributing to change of modal behaviour of the panel. In this study a FEM calculations are used for determining of natural frequencies and mode shapes of sandwich structures designed for production by selective laser sintering technology. Analyses of the obtained results presents base for optimal design of light-weight structures in automotive, aerospace and naval industries.
\end{abstract}

\section{Keywords: Sandwich panels, Modal analysis, Additive manufacturing, Lattice and cellular structures}

\section{INTRODUCTION}

Sandwich panels, comprising thin plates and core between them, are increasingly common structural elements in variety of applications, including vehicle and aircraft panels, aircraft wings, helicopters rotor blades, aircraft fuselages, racing yachts keels, lightweight housings and bulkheads. More than 700 honeycomb core structures for sandwich components were produced in the past 50 years just by one manufacturer [1, 2, 3]. However, sandwich panels with arbitrary core structure are almost impossible to be manufactured by means of traditional manufacturing technologies. The idea of the paper is to consider use of AM technologies for manufacturing of complex lattice and cellular structures that would simultaneously meet two requests: reduction of mass and reduction of vibrations (and consequently noise emission) of housing panels.

Modal behaviour, as characteristic structure property, is determined by material and geometric properties (mass, stiffness and damping) and boundary conditions of the structure. Change of any of those characteristics leads to changes of modal behaviour [4]. High stiffness of a structure is very important for its good resistance to natural vibrations. On the other hand, lightweight design (mass reduction) is requirement that is met by modern designers with increased occurrence. These two requirements, important from the aspect of the modal behaviour of the structure, are contradictory to each other.

The paper presents initial results of research of modal behaviour of sandwich panels with various shapes and density of lattice structures, with the ultimate goal to determine the optimal sub-structure of a panel from the aspect of reduction of vibrations and sound emission. For example, it has been shown [5] that it is possible to reduce noise emission of gearboxes by increasing the structural stiffness and reduction the vibration of panels with large and positive acoustic contribution coefficients.

Sandwich panels consist of two parallel thin plates and a core between them. The parallel plates withstand normal forces, which arise from bending, tension or pressure, and the core withstands shear forces, like in the case of I-beam geometry. The stiffness of the sandwich structure can be increased by appropriate selection of the core structure. In addition, increasing the distance between the parallel plates by the lightweight core increases the second moment of area (and hence the bending stiffness) of the material cross-section with only a small increase in weight. Likewise, changing of wall thicknesses of plates and/or core can significantly change stiffness, masses and modal frequencies of sandwich plates.

For the purpose of analysis of influence of shapes and dimensions of sub-structures (core structure) on vibration behaviour of sandwich panels are used results of modal analysis by FEM.

\section{AM TECHNOLOGIES}

Unlike traditional methods of manufacturing, where product is made by removing or forming of material, additive manufacturing technologies make product by joining successive layers of a material. There are various types of additive manufacturing technologies, using different materials. The AM technology that enables manufacturing of products with mechanical properties comparable to those made by traditional technologies is called "selective laser sintering" (SLS). SLS offers freedom to build quickly complex and freeform parts that are more durable and provide better functionality than other AM technologies. SLS technology uses laser beam directed by optical system to melt plastic or metal powder. The melted powder is cooling and sintering after the 
illumination, thus forming a horizontal cross-section of a 3D object. After sintering, the layer is lowered and a new layer of powder is applied on top of it. The new layer is then melted, thus forming the next section, which is simultaneously joining with the previous layer during the cooling phase. Therefore, during the cooling phase the melted powder is joining in both horizontal and vertical direction. The process is repeated until the 3D object is made. This new technology enables manufacturing of complex structures with cellular structure that have low mass, high stiffness good stress resistance (Fig. 1a). SLS opens many possibilities for lightweight design. EOS, German SLS machine manufacturer, demonstrated ability to produce metal sandwich structures using this technology (Fig. 1b). It means also that it is possible to manufacture various types of sandwich structures of housing panels by SLS technology $[6,7]$. EADS has been testing wind brackets (Fig. 1c) and hinges for engine covers to evaluate technical and commercial feasibility of parts produced by SLS [8, 9]. Considerable savings and weight reduction is gained without endangering bracket functionality.

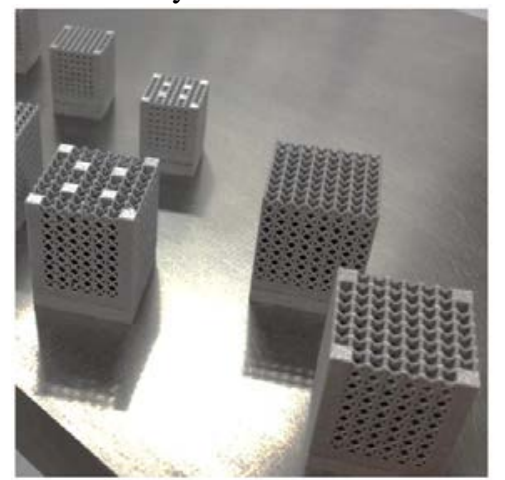

a)

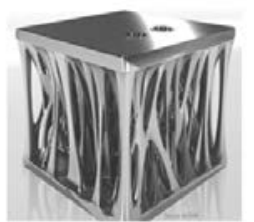

b)

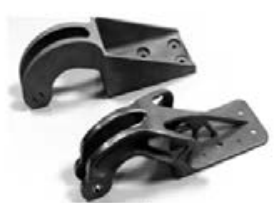

c)
Fig. 1: Lightweight structures manufactured by SLS:

a) Cellular structures [10]; b) Sandwich structure with low core density [12]; c) Titanium bracket with optimized topology [12]

However, SLS technology has also its limitations. The main limitations are connected to limited minimal wall thickness of the parts $(0.4 \mathrm{~mm})$, limited dimensions of the manufactured objects (250x250x330 mm) and limited selection of materials that may be processed by the technology. Available materials are 15-5 Stainless Steel, Maraging Steel, Cobalt Chrome, Titanium Ti64, Nickel Alloy N62 and Aluminium alloys. Parts are being manufactured on building platform, which has role to remove heat and prevent motion and deformation of objects that may occur due to the residual stresses caused by thermal dilatation. The manufactured parts are thus connected to the platform by supports that are manufactured simultaneously with the object. The supports sometimes present an additional limitation in design for additive technologies.

\section{MODELS DEVELOPMENT}

Modelling and analysis of the results are conducted in two stages.

In the first stage were developed twenty-three geometric models of sandwich panels with variable core structure for the purpose of analysis of the influence of substructure shape on natural vibrations of sandwich panels. Widths of the sandwich cores were $h_{c}=13 \mathrm{~mm}$, thicknesses of parallel cover plates were $t_{p}=1 \mathrm{~mm}$ and thicknesses of core walls were $t_{c}=1 \mathrm{~mm}$ (Fig. 2).

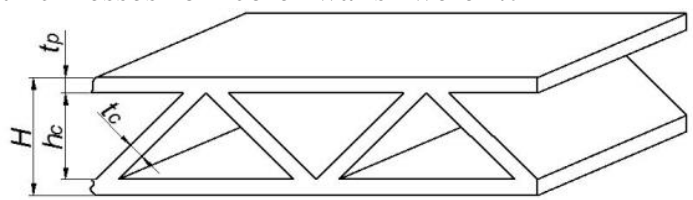

Fig.2: Sandwich panel structure

Models were made using Solid Works software package. Three different methods were used for modelling of 3D models of substructures. The first method was extrusion of a 2D sketch to 3D solid model (Fig. 3a). The second method was application of Boolean operations to obtain the model by adding or subtracting primitives (Fig. 3b). The third method was multiplication of 3D solid blocks obtained by extrusion of a 2D sketch (Fig. 3c).

a)
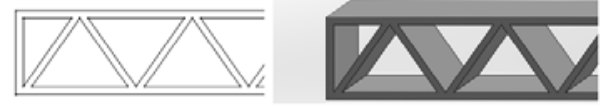

b)

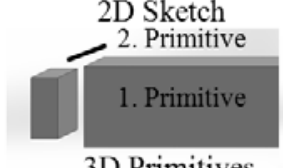

Extruded 3D Solid

3D Primitives

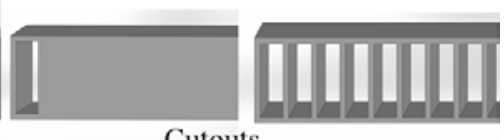

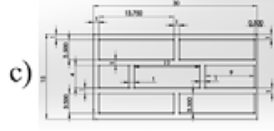

2D Sketchng

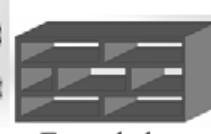

Extruded Solid block
Cutouts

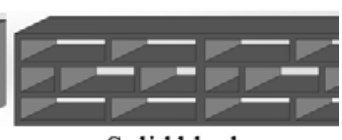

Solid blocks
Fig. 3: Illustration of different methods for modelling of sandwich panels

All of the panels had the same length and heights 300x200 mm. The solid block with the same dimensions would have density of $7850 \mathrm{~kg} / \mathrm{m}_{3}$ and its mass would be $7.065 \mathrm{~kg}$. Densities of the sandwich panels varied from $1823.33 \mathrm{~kg} / \mathrm{m}_{3}$ to $4338.88 \mathrm{~kg} / \mathrm{m}_{3}$, and the masses varied from $1.641 \mathrm{~kg}$ to $3.905 \mathrm{~kg}$. The developed models of sandwich panels are presented in [11].

The second phase of research continues on selected models, which showed the highest values of natural frequencies, after the modal analysis. These are the models V2, V12 i Vhc (table 1). New variants of the models were developed with variation of plate thickness, core wall thickness and core width and calculation of natural frequencies for each variation of the sandwich panel. The plate thickness was varied from $1 \mathrm{~mm}$ to $4 \mathrm{~mm}$ and the core wall thickness was varied from $1 \mathrm{~mm}$ to $3 \mathrm{~mm}$, all with $1 \mathrm{~mm}$ steps. The core widths were selected to provide sandwich panel widths of $15 \mathrm{~mm}, 20 \mathrm{~mm}$ and $25 \mathrm{~mm}$.

\section{MODAL ANALYSIS}

Modal analysis represents the first step in research of vibrations of mechanical systems. Natural frequencies and modal shapes of the studied types of sandwich panels were determined using software package ANSYS. 
Table 1: Sandwich panels with minimal number of calculated modes

\begin{tabular}{|c|c|c|c|c|c|c|c|c|c|}
\hline Ver. & $\begin{array}{c}\text { Sandwich structure } \\
\text { design }\end{array}$ & $\begin{array}{c}\text { Mass } \\
\mathrm{kg}\end{array}$ & $\begin{array}{l}\text { Density } \\
\mathrm{kg} / \mathrm{m}^{3}\end{array}$ & $\begin{array}{c}\text { No } \\
\text { of } \\
\text { modal } \\
\text { shapes }\end{array}$ & Ver. & $\begin{array}{c}\text { Sandwich structure } \\
\text { design }\end{array}$ & $\begin{array}{c}\text { Mass } \\
\mathrm{kg}\end{array}$ & $\begin{array}{l}\text { Density } \\
\mathrm{kg} / \mathrm{m}^{3}\end{array}$ & $\begin{array}{c}\text { No } \\
\text { of } \\
\text { modal } \\
\text { shapes }\end{array}$ \\
\hline V2 & & 2.16 & 2400.0 & 7 & V19 & & 2.84 & 3155.5 & 7 \\
\hline V12 & & 1.68 & 2866.6 & 7 & V21 & & 3.59 & 3988.8 & 7 \\
\hline V14 & & 3.91 & 4333.3 & 7 & V2.1 & & 2.72 & 3022.2 & 7 \\
\hline V15 & & 3,16 & 3511.1 & 7 & V2.2 & & 2.72 & 3022.2 & 7 \\
\hline $\begin{array}{c}\text { Solid } \\
\text { model }\end{array}$ & & 7.06 & 7850 & 6 & Vhc & & 2.11 & 2344.4 & 7 \\
\hline
\end{tabular}

The discretized model of a sandwich panel is shown in Fig. 4. Boundary conditions are defined by fixing nodes at the bottom side of panels (Fig. 4).

Frequency range for modal analysis was from $0 \mathrm{~Hz}$ to $3000 \mathrm{~Hz}$. The assumed material of the models in the presented modal analysis was steel.

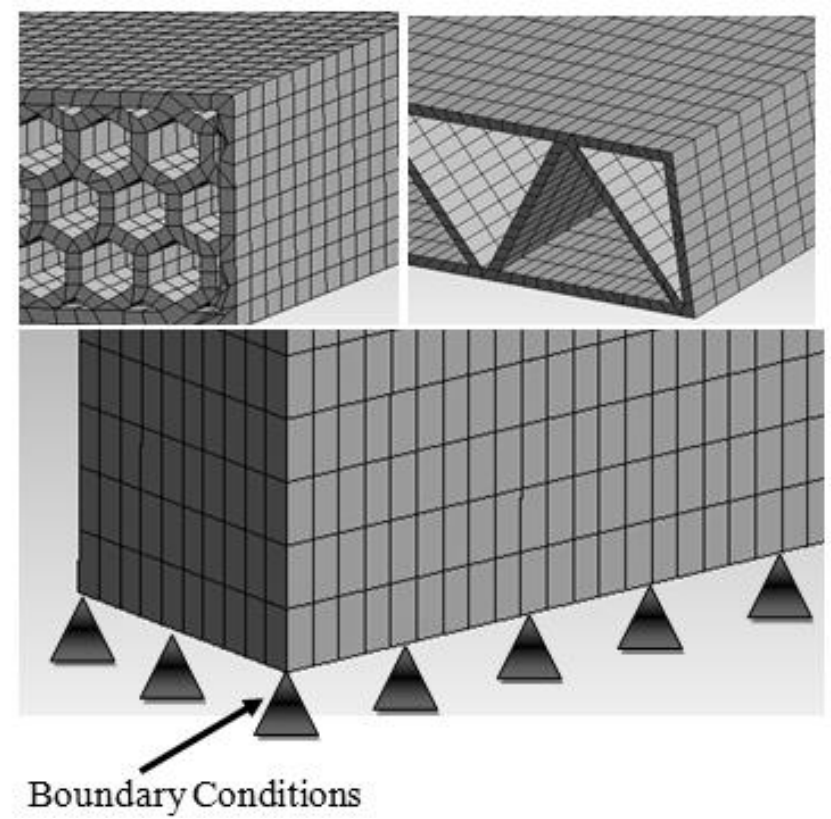

Fig. 4: The discretized model of a sandwich panel

\section{RESULTS AND DISCUSION}

For solid model of panel were determined six natural frequencies and modal shapes of vibrations within the studied frequency range. The number of determined vibration modes of sandwich panels developed in the first stage was between 7 and 12 in the studied frequency range. Modal shapes for the model V12 are presented in Fig 5. The smallest number of possible modes of oscillation is obtained for sandwich panels marked as: V2, V12, V14, V15, V19, V20, V21, V2.1, V2.2, Vhc which are presented in Table 1. Values of the modal frequencies of these models are presented in Fig.6. Modal shapes of model V12 are presented in Fig.5. A brief analysis of the results obtained during the first stage shows that the solid model has the highest density and the lowest natural frequencies (Fig. 6) of all studied models.

The conclusion which it can be draw from obtained results is that the sandwich panels have substantially lower weights and wider operational bandwidths than solid panels, which justifies the effort to replace solid panels by sandwich panels. The Fig. 6 shows that the highest modal frequencies are obtained for the models V2, V12 i Vhc. On the other hand, the corresponding amplitudes of natural vibrations of solid panel are the smallest, as expected (Fig.7). That means that all aspects of vibration behaviour of sandwich panels need careful consideration before a selection is made for a specific application.

Since the models V12 and Vhc had the lowest mass, and also belong to the group of models with the smallest number of natural vibration modes with frequencies in the considered range, they were selected to be the object of the analyses in the second stage of the research. 

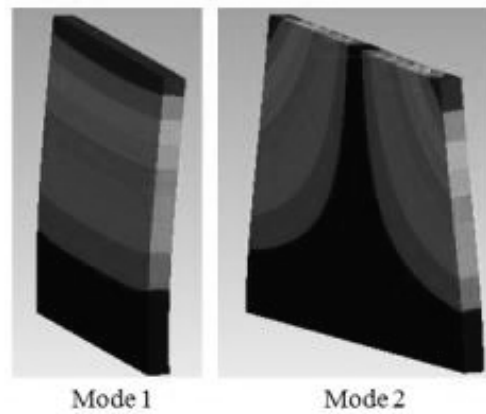

Mode 2

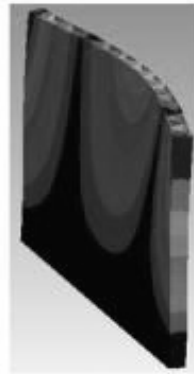

Mode 3

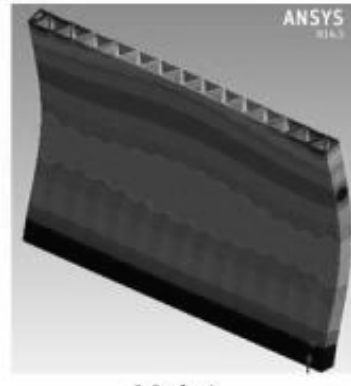

Mode 4

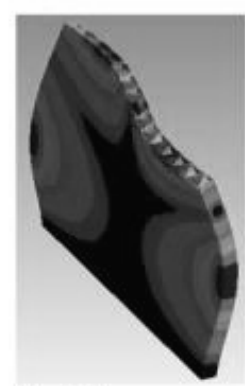

Modes

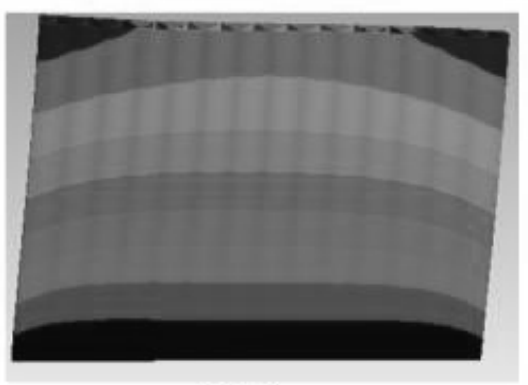

Mode 6

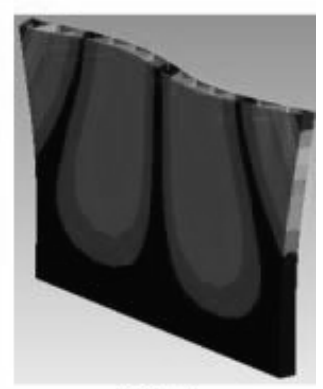

Mode 7

Fig.5: Modal shapes of a sandwich panel - model V12

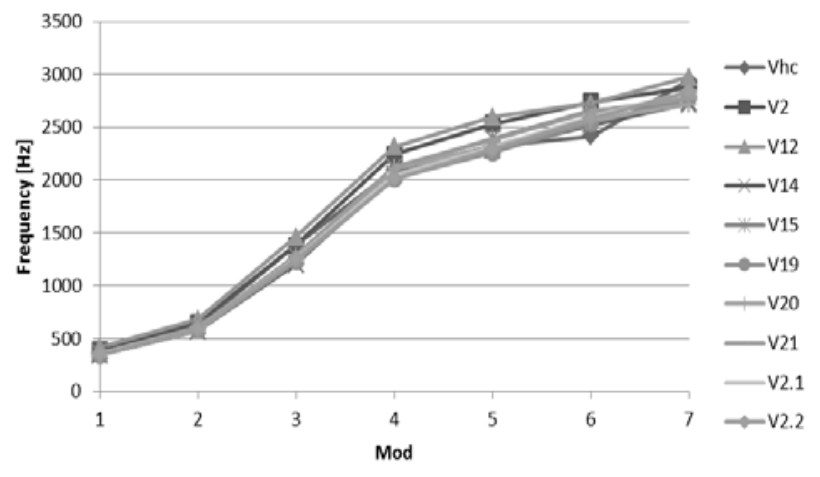

Fig. 6: Diagram of the natural frequencies

According to the previously described plan of research were developed models of V12 sandwich panels with variable plate thickness, core wall thickness and core width. Natural frequencies of all the models were calculated using the ANSYS software package and the results are presented in Fig. 8-Fig. 10.

In the Fig. 8, on an example of the model V12 is shown dependency of the natural frequencies on:

- core wall thickness, where plate thickness was kept constant (shown in the diagram on the left);

- plate thickness, where core wall thickness was kept constant (shown in the diagram in the middle);

- thicknesses of both core wall and plate, which were kept equal (shown in the diagram on the right);

In all the considered cases panel width was kept constant at $H=15 \mathrm{~mm}$.

The presented diagrams show that:

- the increase of the core wall thickness leads to decrease of natural frequencies of the structure;

- natural frequencies of the structure are the highest when the plates are twice thicker than the core walls;

- natural frequencies decrease if both the core wall thickness and the plate thickness are equally increased;

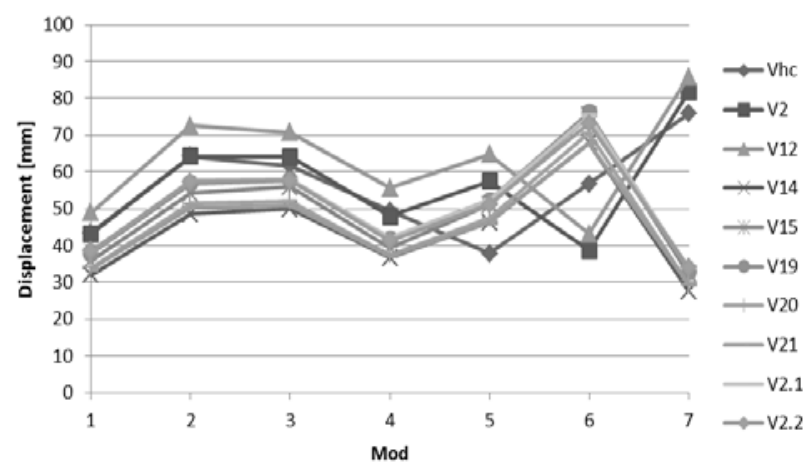

Fig. 7: Diagram of the maximal displacements

The observed existence of maximum in dependence of natural frequencies on the ratio between plate thickness and core wall thickness was further studied by analyzing the effects of variation of the panel width. The results of the analyses are shown in the Fig. 9, which shows that the increase of the panel width leads to decrease of number of natural vibration modes in the considered frequency range. The number of natural vibration modes is six or even less (depending on the plate thickness and core width). For the models with panel width $H=15 \mathrm{~mm}$ is six, five for the models with panel width $H=20 \mathrm{~mm}$, and only four for the models with panel width $H=25 \mathrm{~mm}$. However, natural frequencies of all the natural vibration modes had the highest value when the plates were twice thicker than the core wall.

The influence of core width to the frequencies of natural vibrations was also studied and the results are presented in the Fig. 10, which shows that the frequencies of natural vibration modes increase with increase of the core width.

The changes of all geometric parameters of a sandwich structure influence its mass and density, and thus also to amplitudes of its natural vibrations. In general, the corresponding amplitudes decrease with increase of 
density, which is presented in the Fig. 11, where these two

quantities are shown in a log-log diagram.

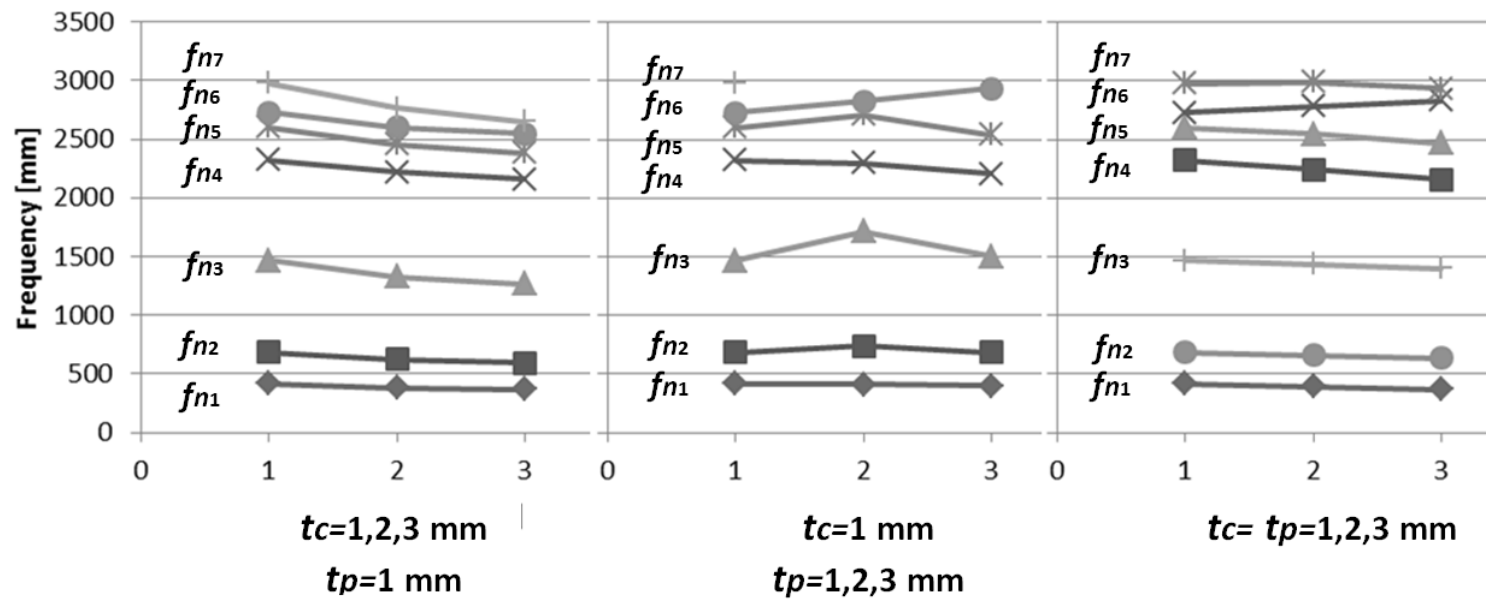

Fig. 8: Dependence of natural frequencies on core wall thickness (tc) and plate thickness (tp) for sandwich panels with width $H=15 \mathrm{~mm}$
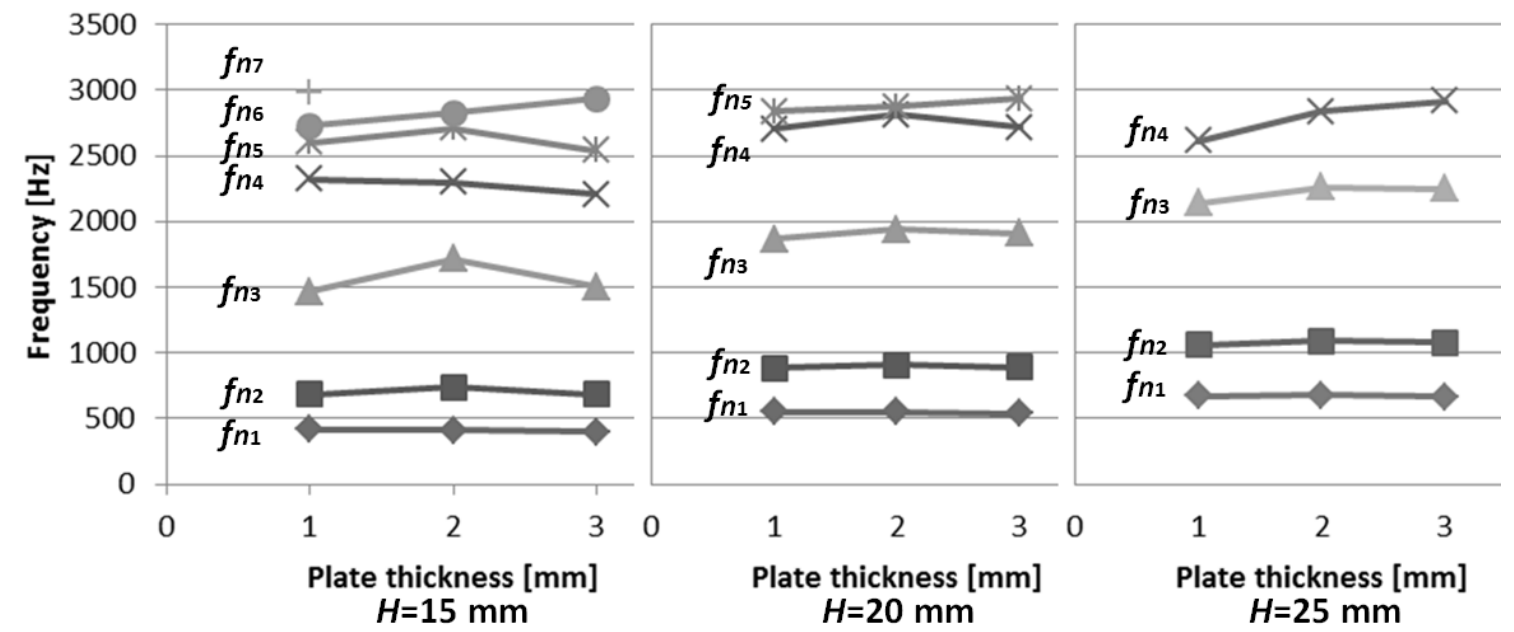

Fig. 9: Dependence of natural frequencies on plate thickness ( $t p=1,2,3 \mathrm{~mm})$ and panel width $(H)$ for constant value of core wall thickness $t c=1 \mathrm{~mm}$

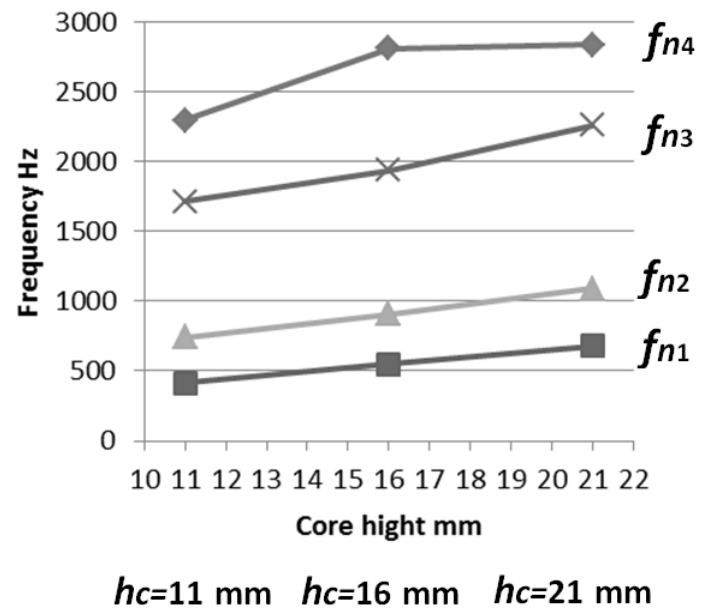

Fig.10: Dependence of natural frequencies on core width (hc) for core wall thickness tc $=1 \mathrm{~mm}$ and plate thickness $t p=2 \mathrm{~mm}$

The slope of the approximate line is $-1 / 2$, which indicates that the amplitude of oscillation is proportional to the inverse square root density. The figure shows that lowfrequency modes obey the inverse-square-root dependence for all samples, which are shown in Fig. 10. On the other hand, high-frequency modes show significant deviations from the inverse-square-root dependence. It means that the shape of the substructure of the core influences the amplitudes of natural vibrations at high frequencies.

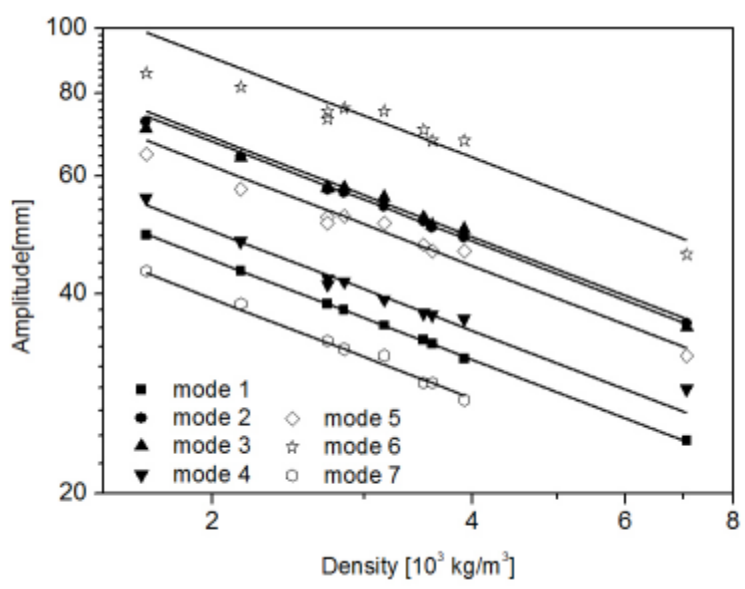

Fig. 11: Dependence of the corresponding amplitudes on the density of the sandwich panels

Such behaviour may be explained by ratio between the wavelengths of vibrations and characteristic dimensions of the substructures. When the wavelengths of vibrations are large in comparison with characteristic 
dimensions of the substructure, then the whole volume of the substructure cells is subjected to approximately constant stress, as it is the case with solid panels. Therefore, the differences between shapes of the substructures have no influence on the amplitudes of lowfrequency vibrations. On the other hand, when the wavelengths of vibrations are small, then various parts of the substructure cells are subjected to various stresses, and the shape of the substructure influences the amplitudes of the vibrations.

\section{CONCLUSON}

The presented research considered influence of geometric parameters on modal behavior of sandwich panels. Modal behaviour of twenty-three types of sandwich panels was studied by numerical methods. Two models with the lowest density, mass, and the lowest number of possible natural frequencies of the observed frequency range of $0-3000 \mathrm{~Hz}$ have been identified. The further study was consisted in analyses of influence of plate thickness, core wall thickness and panel width to number of modes that can be excited, frequencies of natural vibration modes and amplitude levels of the sandwich panel.

Obtained results have shown that the natural frequencies of the sandwich panel have the highest natural frequencies when the plates are twice thicker than core walls. The dependency is more pronounced with thin sandwich panels and panels with thin core walls.

Increase of core wall thickness leads to decrease, and increase of the core high leads to increase of the natural frequencies of sandwich panels.

The results also confirmed previous results [11] that the amplitudes of the natural vibrations depend on the shape of substructure when the wavelength of the vibrations is comparable to characteristic dimensions of the substructure.

\section{ACKNOWLEDGEMENT}

The authors wish to express their gratitude to Ministry for education, science and technology of Republic of Serbia for support through research grants TR37020 and TR35006.

\section{REFERENCES}

[1] Kopp, G., Kuppinger, J., Friedrich, H.E., Henenning, F.: "Innovative Sandwich Structures for Functionally Integrated Lightweight Design", ATZ worldwide, Volume 111, Issue 4, pp. 44-49, 2009.

[2] Nilsson, E., Nilsson, A.C.: "Prediction and measurement of Some Dynamic properties of Sandwich Structures with Honeycomb and Foam Cores", Journal of Sound and Vibration, 251(3), pp. 409-430, 2002.

[3] Harish, R., Sharma, R.S.: "Vibration Response Analysis of Honeycomb Sandwich Panel with Varying Core Height”, International Journal of Emerging Technologies in Computational and Applied Sciences (IJETCAS), 13-433, pp. 582-586, 2013.

[4] Ćirić-Kostić, S., Ognjanović, M.: "The Noise Structure of Gear Transmission Units and the Role of Gearbox Panels." FME Transactions 35 (2), pp. 105-112, 2007.

[5]. Zhou, J., Sun, W., Tao, Q.: “Gearbox Low-Noise Design Method Based on Panel Acoustic Contribution”, Mathematical Problems in Engineering, vol. 2014, Article ID 850549, 10 pages, 2014.

[6] McCarthy, D.L., Williams, C.B.: " Creating Complex Hollow Metal Geometries Using Additive Manufacturing and Electroforming ", International Solid Freeform Fabrication Symposium, pp. 108-120, 2012.

[7] Suman, D. et al. "Direct Laser |Freeform Fabrication of High Performance Metal Components." Rapid Prototyping Journal 4.3, pp.112-117, 1998.

[8] Tomlin, M., Meyer, J.: "Topology Optimization of an Additive Layer Manufactured (ALM) Aerospace Part." The 7th Altair CAE Technology Conference, pp.1-9, 2011.

[9] Additive manufacturing - Strategic Research Agenda 2014, AM Platform, 2014.

[10] Campanelli, S.L., Contuzzi, N., Ludovico, A.D., Caiazzo, F., Cardaropoli, F., Sergi, V.: "Manufacturing and Characterization of Ti6Al4V Lattice Components Manufactured by Selective Laser Melting", Materials, 7, pp.4803-4822, 2014.

[11] Vranić, A., Ćirić-Kostić, S., Tatić, B.: "Influence of Sub-Structures' Shape on Vibration Behaviour of Sandwich Walls", Proceedings of the VIII International Conference Heavy Machinery - HM 2014, pp. E17-22, Kraljevo, Serbia, 2014.

[12] www.eos.info 